\title{
Facile Synthesis of Well-Defined Block Copolymers Containing Regioregular Poly(3-hexyl thiophene) via Anionic Macroinitiation Method and Their Self-Assembly Behavior
}

\author{
Chi-An Dai, ${ }^{*},+, \ddagger$ Wei-Che Yen, ${ }^{\dagger}$ Yi-Huan Lee, ${ }^{\dagger}$ Chun-Chi Ho, ${ }^{\S}$ and Wei-Fang Su, ${ }^{*}, \uparrow \S$ \\ Institute of Polymer Science and Engineering, Department of Chemical Engineering, and Department of Materials \\ Science and Engineering, National Taiwan University, Taipei, 106 Taiwan \\ Received May 14, 2007; E-mail: polymer@ntu.edu.tw; wfsu@ntu.edu.tw
}

\begin{abstract}
The self-assembly of block copolymers into a nanostructure with novel morphology and property has attracted an increasing interest as a new approach for materials science, chemical synthesis, and nanofabrication. ${ }^{1,2}$ Most theoretical and experimental studies have concentrated on the assembly characteristics of coil-coil block copolymers. Recently, rod-coil block copolymers have received a great deal of attention since they offer an attractive strategy for the organization of many highly functional rod-like polymers such as helical biopolymers and conducting polymers with rigid $\pi$-conjugated backbones. ${ }^{1,3-9}$ In particular, block copolymers containing conducting polymer segments, such as polyfluorene, poly(phenylene vinylene), and polythiophene, are technologically important since these homopolymers may be used in low cost, large area, and flexible electronics. ${ }^{1,10-17}$ For example, in organic electronic devices such as photovoltaic cells, their efficiency depends critically on charge separation and transport in the nanostructures of the devices. Conducting block copolymers with highly ordered heterogeneous nanostructure may provide an enhanced interfacial area for charge separation as well as an efficient pathway for charge transport. ${ }^{18}$ In addition, it was shown that integrating a rod-like conducting polymer in copolymer structures with various coil-like polymer blocks displays better mechanical properties which provide the desirable processing property for novel organic optoelectronic device fabrication. ${ }^{16,19}$
\end{abstract}

In order to create a highly ordered nanostructure from these structure-directing block copolymers, the synthesis method to precisely control the molecule weight and distribution for each block is greatly needed. Recently, it was shown that coupling of endfunctionalized monodispersed homopolymers may provide a facile synthesis technique for the formation of blocks with novel copolymer nanostructures, while retaining information and control over the length distribution of the individual blocks. ${ }^{15,20-22}$ However, end-end coupling reactions are often hindered by the chain length effect of end-functionalized homopolymers, leading to a decrease in the coupling efficiency. ${ }^{23}$ The most widely used technique for the preparation of block copolymers is living anionic polymerization, which often involves sequential addition of vinylic monomers. For block copolymers involving a conducting polymer and a vinylic polymer, a combination of polymerization methods is needed as the conducting polymers are often synthesized using the polycondensation method. Among various conducting polymers, regioregular poly(3-hexyl thiophene)s (P3HT) are widely studied because of their superior optoelectonic property and chemical stability. McCullough et al. and Yokozawa et al. discovered that well-defined end-functionalized P3HTs can be synthesized with low polydis-

\footnotetext{
$\dagger$ Institute of Polymer Science and Engineering.

Department of Chemical Engineering.

$\S$ Department of Materials Science and Engineering.
}

Scheme $1^{a}$

(a)

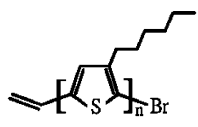

1

(b)

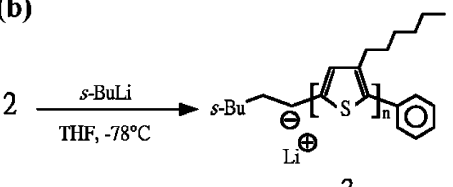

3
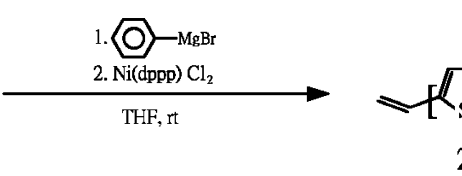

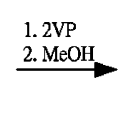

${ }^{a}$ Conditions: (a) synthesis scheme of $\alpha$-vinyl- $\omega$-(phenyl) end-functionalized P3HT; (b) $\alpha$-vinyl- $\omega$-(phenyl) end-functionalized P3HT is activated by $s$-BuLi and forms $\mathbf{3}$, which subsequently serves as an anionic macroinitiator for the synthesis of P3HT-P2VP; dppp = propane-1,3-diylbis(diphenylphosphane).

persity via a chain growth mechanism by using a catalyst-transfer polycondensation method mediated by a nickel catalyst (GRIM method). ${ }^{24-26} \mathrm{McC}$ cullough et al. further demonstrated that P3HT block copolymers can be synthesized from a linker molecule attached to an end-functionalized $\mathrm{P} 3 \mathrm{HT}$ as a macroinitiator via atom transfer radical polymerization (ATRP). ${ }^{13,16}$

In this communication, we demonstrate that a vinyl endterminated P3HT can be activated at the terminal double bond with $s e c$-butyllithium ( $s$-BuLi) for subsequent anionic polymerization. The resulting poly(3-hexyl thiophyllithium) (3) can then be used as a living anionic macroinitiator for the polymerization of 2-vinyl pyridine (2VP) monomers to form monodispersed P3HT-P2VP diblock copolymers with high yield. This combination synthesis method takes advantage of the fact that both the GRIM method synthesized P3HTs have well-defined chain ends and their activated macroinitiator anions are stabilized by their conjugated segments yet they are strong enough to initiate 2VP for the synthesis of welldefined block copolymers. Different block ratio of P3HT to P2VP can be synthesized by changing the molar ratio of $2 \mathrm{VP}$ to P3HT. The chemical structure and the self-assembly behavior of the block copolymers were studied by various techniques such as NMR, GPC, TEM, and SAXS. We showed that these conducting-insulating block copolymers are able to undergo microphase separation and self-assemble into nanostructures of sphere, cylinder, lamellae, and nanofiber structure with increasing rod ratios. Both the synthesis method that employs conducting macroinitiator for anionic polymerization and the block copolymers thus synthesized are unprecedented.

We synthesize the diblock copolymer by first synthesizing homopolymer P3HT (1), which is chain-end-functionalized with a vinyl group at the $\alpha$ position (Scheme 1a). The synthesis of $\mathbf{1}$ from the 2,5-dibromo-3-hexylthiophene monomer and the vinyl end- 


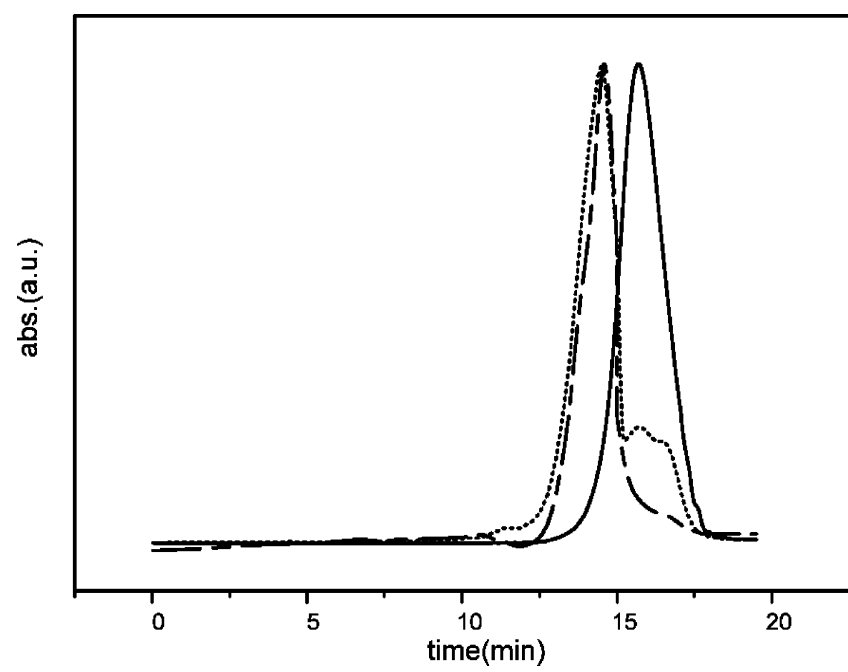

Figure 1. GPC traces of (-) homopolymer poly(3-hexyl thiophene), $(--)$ as synthesized P2 block copolymer $\left(f_{\mathrm{P} 3 \mathrm{HT}}=0.25\right)$ before solvent extraction, and (- -) P2 after solvent extraction to remove uninitiated poly(3-hexyl thiophene) homopolymer.

capping reaction with high capping yield ( $90 \%)$ was conducted according to the literature procedure..$^{25,27} \mathbf{1}$ was further $\omega$ end-capped with a phenyl group by the reaction with Grignard reagent phenylmagnesium bromide (see Supporting Information). This $\omega$ end-capping reaction was performed to prevent lithium-halogen exchange of $\mathbf{1}$ with $s$-BuLi subsequently added for anionic polymerization, leading to different lithiations for initiation at the two ends of P3HT. ${ }^{28}$ The resulting $\alpha$-vinyl- $\omega$-(phenyl) end functional P3HT (2) was well-characterized with gel permeation chromatography (GPC) and ${ }^{1} \mathrm{H}$ NMR, and the same P3HT was used for all subsequent diblock copolymer syntheses. The numberaveraged molecular weight and polydispersity index of $\mathbf{2}$ are 7000 and 1.17, respectively. Completion of the end substitution reactions was confirmed by the appearance of a vinyl signal $(\delta 5.12$ and $5.51)$ and a phenyl signal $(\delta 7.43-7.47)$ in the ${ }^{1} \mathrm{H}$ NMR (see Supporting Information).

The schematic for synthesizing P3HT-P2VP block copolymer is shown in Scheme 1b. 2 was first dissolved in THF, and the solution was cooled to $-78{ }^{\circ} \mathrm{C}$ for anionic polymerization. 2 was then initiated with the addition of an excess amount of $s$-BuLi (4-5 molar equiv to 2) to ensure the formation of poly(3-hexyl thiophyllithium) macroinitiator. We found that poly(3-hexyl thiophyllithium) (3) in THF is stable at a temperature of $40{ }^{\circ} \mathrm{C}$, while the excess amount of $s$-BuLi can readily react with THF at elevated temperature, forming ethylene and alkoxides. ${ }^{29}$ The solution was kept at $40{ }^{\circ} \mathrm{C}$ for 15 to $30 \mathrm{~min}$ to completely remove the excess $s$-BuLi and then cooled back to $-78^{\circ} \mathrm{C}$. $2 \mathrm{VP}$ monomers were then added into the $\mathbf{3}$ macroinitiator solution for initiation and polymerization. The reaction proceeded for $3 \mathrm{~h}$, and a drop of degassed methanol was then added to the solution to terminate the polymerization. The solution was then precipitated with hexane. The precipitant was redissolved in THF/hexane $(2: 3, \mathrm{v} / \mathrm{v})$. Pure block copolymer can be separated from the copolymer/homopolymer mixture by using $\mathrm{MeOH} /$ water $(3: 1, \mathrm{v} / \mathrm{v})$ as an extraction solvent from the THF/hexane solution.

Completion of the reaction was determined by the disappearance of the vinyl signals in the ${ }^{1} \mathrm{H}$ NMR spectra (see Supporting Information). Furthermore, for performed anionic polymerizations, a major shift in the GPC trace was observed toward higher molecular weight, indicating block copolymer formation (dotted line in Figure 1). This major peak with narrow distribution in the
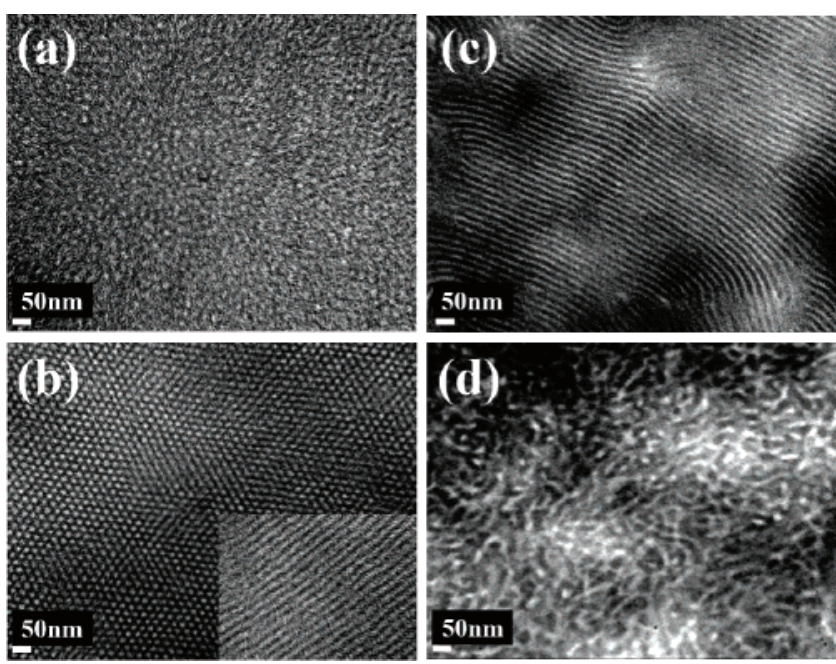

Figure 2. TEM pictures of the self-assembled structure of (a) P1 (spheres or disorder micelles), (b) P2 (hexagonal close-packed cylinders), (c) P3 (lamellae), and (d) P4 (nanofibers). The inset in (b) shows the side view of HCP.

GPC trace shows evidence of a living chain growth mechanism involving the anionic polymerization of $2 \mathrm{VP}$ initiated by P3HT macroinitiator 3. In addition, a minor peak with an average elution time appears at the same location as that for the $\mathrm{P} 3 \mathrm{HT}$ homopolymer 2, indicating some uninitiated or terminated P3HT macroinitiator (Figure 1). This minor peak in GPC appeared in all copolymer synthesis accounts for less than $20 \%$ of the initial P3HT. Subsequently, this unreacted P3HT can be removed by using the extraction method described above. The GPC trace for the block copolymer P3HT-P2VP synthesized after the extraction is shown in Figure 1. After purification, the polydispersity index and the weight fraction of the block copolymer P3HT-P2VP were evaluated by GPC and ${ }^{1} \mathrm{H}$ NMR, respectively. Four P3HT-P2VP diblock copolymers, referred to as $\mathrm{P} 1, \mathrm{P} 2, \mathrm{P} 3$, and $\mathrm{P} 4$, were synthesized for this study. The polydispersity indices for all block copolymers are less than 1.3 , and their P3HT wt $\%, f_{\mathrm{P} 3 \mathrm{HT}}$, are $14,25,41$, and 70 , respectively.

We also report on the first structural investigations of the P3HTP2VP diblock copolymers employing transmission electron microscopy and small-angle X-ray scattering. For both measurements, bulk samples were prepared by slowly evaporating the copolymer solutions in THF for 3 days at room temperature followed by annealing the sample in a high vacuum oven $\left(10^{-6}\right.$ Torr $)$ at $130^{\circ} \mathrm{C}$ for 3 days. The bulk samples were then microtomed, and the cut film with thickness $<100 \mathrm{~nm}$ was stained with iodine for the P2VP block for TEM observation. Figure 2 shows disorder micelles or spheres (S), hexagonal close-packed cylinders (HCP), lamellae (L), and filament-like or nanofiber structure observed for P1, P2, P3, and $\mathrm{P} 4$, respectively. For $\mathrm{P} 1-\mathrm{P} 3$, their morphology is similar to that of a coil-coil counterpart roughly at a similar block ratio. However, with increasing P3HT fraction from 14 to $41 \%$, there is a strong tendency for P3HT-PVP to self-organize into highly ordered structures (HCP and L). Furthermore, for P4, the copolymer with the highest weight fraction of $\mathrm{P} 3 \mathrm{HT}$, it appears that the strong rod-rod interaction between regioregular P3HT in the copolymer suppresses the long-range order of the nanostructure, and nanofiber morphology is observed.

The microstructure and domain spacing for copolymers $\mathrm{P} 1-\mathrm{P} 4$ were further examined using small-angle X-ray scattering technique (SAXS). A representative SAXS pattern obtained for P1 is shown in Figure 3a. The main peak is centered around a value for the 


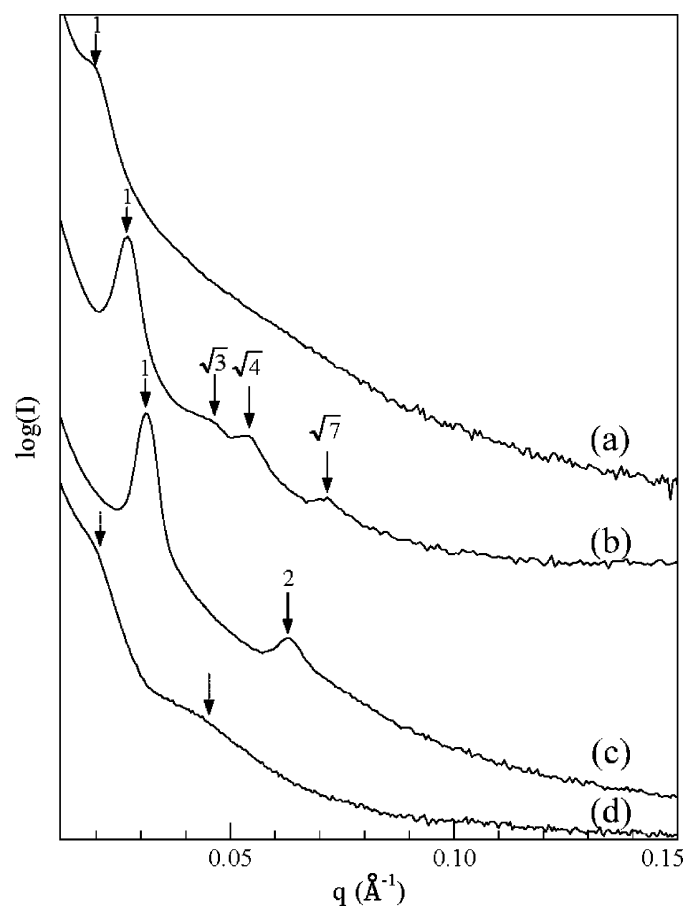

Figure 3. $\mathrm{SAXS}$ spectra for (a) $\mathrm{P} 1\left(f_{\mathrm{P} 3 \mathrm{HT}}=0.14\right)$, (b) $\mathrm{P} 2\left(f_{\mathrm{P} 3 \mathrm{HT}}=0.25\right)$, (c) $\mathrm{P} 3\left(f_{\mathrm{P} 3 \mathrm{HT}}=0.41\right)$, and $(\mathrm{d}) \mathrm{P} 4\left(f_{\mathrm{P} 3 \mathrm{HT}}=0.70\right)$.

scattering wave vector $q$ corresponding to $\sim 30 \mathrm{~nm}$. No higher order reflections are visible on the spectrum. This pattern is consistent with disordered micelles (or spheres) and consistent with the TEM observation. In the SAXS pattern of P2 (Figure 3b), the main peak is located at a $q$ value corresponding to $\sim 23.3 \mathrm{~nm}$, and there are higher order reflections at angular positions of $\sqrt{3}, \sqrt{4}$, and $\sqrt{7}$ of this first-order maximum. This spacing is consistent with a HCP and consistent with the TEM observation. For P3 (Figure 3c), the main peak is centered around a $q$ value corresponding to $\sim 19.3$ $\mathrm{nm}$, and there is one higher order reflection clearly visible at an integral multiple 2 of this $q$ value. Such a sequence is consistent with an arrangement of lamellae and consistent with the TEM measurement. For P4, there are two main but broad peaks. The center of the first main peak is located at a $q$ value corresponding to roughly $\sim 32 \mathrm{~nm}$, which corresponds to roughly the lateral spacing of the nanofiber observed in TEM. The nanofiber structure was also observed in a similar block copolymer system synthesized using the ATRP method by McCullough et al. They found that, for longer P3HT block $\left(M_{\mathrm{n}}>15000\right)$, all block copolymers exhibit predominately nanofiber structure even for small P3HT fraction $\left(f_{\mathrm{P} 3 \mathrm{HT}} \sim 15 \%\right)$ in the copolymer chain. ${ }^{13}$ In our system, the P3HT is shorter $\left(M_{\mathrm{n}} \sim 7000\right)$, and the copolymer exhibits nanofiber structure only until the P3HT weight fraction is $>70 \%$. For P3HT, with a weight fraction $<50 \%$, we have found that the P3HT-P2VP block copolymers exhibit conventional coil-coil nanostructures.

In conclusion, we have described a novel method to use an endfunctionalized conducting polymer as an anionic macroinitiator for the preparation of a new set of conducting-insulating diblock copolymers consisting of P3HT and P2VP. We demonstrated that these block copolymers microphase separate and self-assemble into nanostructures of sphere, cylinder, lamellae, and nanofiber structures according to different P2VP volume fractions. The synthesis combines two efficient reactions, the Grignard metathesis method of P3HT and anionic polymerization of 2-vinyl pyridine from the activated vinyl-terminated P3HT macroinitiator, and leads to block copolymers with well-defined molecular weight and narrow molecular weight distribution. It opens up the possibility of a combinatorial approach to the synthesis of novel rod-coil block copolymers.

Acknowledgment. The financial support of this work from the National Science Council of Taiwan (NSC 95-2120-M-002-004) and U.S. Air Force (AOARD-064044) is greatly appreciated. The authors also thank Dr. U-Ser Jeng for his help on the SAXS experiments at the National Synchrotron Radiation Research Center of Taiwan.

Supporting Information Available: Detailed synthesis procedure and characterization of $\mathbf{1}, \mathbf{2}$, and $\mathbf{4}$. This material is available free of charge via the Internet at http://pubs.acs.org.

\section{References}

(1) Jenekhe, S. A.; Chen, X. L. Science 1998, 279, 1903

(2) Klok, H. A.; Lecommandoux, S. Adv. Mater. 2001, 13, 1217

(3) Li, W. J.; Maddux, T.; Yu, L. P. Macromolecules 1996, 29, 7329

(4) Loos, K.; Muller, A. H. E. Biomacromolecules 2002, 3, 368.

(5) Rahman, M. S.; Samal, S.; Lee, J. S. Macromolecules 2006, 39, 5009.

(6) Pryamitsyn, V.; Ganesan, V. J. Chem. Phys. 2004, 120, 5824.

(7) Leclere, P.; Calderone, A.; Marsitzky, D.; Francke, V.; Geerts, Y.; Mullen, K.; Bredas, J. L.; Lazzaroni, R. Adv. Mater. 2000, 12, 1042-1046.

(8) Olsen, B. D.; Segalman, R. A. Macromolecules 2006, 39, 7078-7083.

(9) Kong, X.; Jenekhe, S. A. Macromolecules 2004, 37, 8180.

(10) Marsitzky, D.; Brand, T.; Geerts, Y.; Klapper, M.; Mullen, K. Macromol. Rapid Commun. 1998, 19, 385.

(11) Hempenius, M. A.; Langeveld-Voss, B. M. W.; van Haare, J. A. E. H. Janssen, R. A. J.; Sheiko, S. S.; Spatz, J. P.; Moller, M.; Meijer, E. W. J. Am. Chem. Soc. 1998, 120, 2798-2804

(12) Li, W. J.; Wang, H. B.; Yu, L. P.; Morkved, T. L.; Jaeger, H. M. Macromolecules 1999, 32, 3034.

(13) Liu, J. S.; Sheina, E.; Kowalewski, T.; McCullough, R. D. Angew. Chem., Int. Ed. 2002, 41, 329.

(14) Zhao, Y. L.; Higashihara, T.; Sugiyama, K.; Hirao, A. J. Am. Chem. Soc. 2005, 127, 14158 .

(15) Olsen, B. D.; Segalman, R. A. Macromolecules 2005, 38, 10127

(16) Iovu, M. C.; Jeffries-El, M.; Sheina, E. E.; Cooper, J. R.; McCullough, R. D. Polymer 2005, 46, 8582.

(17) Li, B.; Sauve, G.; Iovu, M. C.; Jeffries-EL, M.; Zhang, R.; Cooper, J.; Santhanam, S.; Schultz, L.; Revelli, J. C.; Kusne, A. G.; Kowalewski, T.; Snyder, J. L.; Weiss, L. E.; Fedder, G. K.; McCullough, R. D.; Lambeth, D. N. Nano Lett. 2006, 6, 1598

(18) Sivula, K.; Ball, Z. T.; Watanabe, N.; Frechet, J. M. J. Adv. Mater. 2006, $18,206$.

(19) Cho, G.; Jung, M.; Yang, H.; Song, J. H.; Sung, M. M. Lamgmuir 2006, $22,4896$.

(20) Opsteen, J. A.; van Hest, J. C. M. Chem. Commun. 2005, 57.

(21) Cotiuga, I.; Picchioni, F.; Agarwal, U. S.; Staal, B. B. P.; Vekemans, J. A. J. M.; Lemstra, P. J. Macromol. Rapid Commun. 2006, 27, 242.

(22) Li, K.; Guo, H.; Liang, Z. Q.; Thiyagarajan, P.; Wang, Q. J. Polym. Sci. Part A: Polym. Chem. 2005, 43, 6007.

(23) Maliakal, A.; Greenaway, H.; O', Shaughnessy, B.; Turro, N. J. Macromolecules 2003, 36, 6075 .

(24) Iovu, M. C.; Sheina, E. E.; Gil, R. R.; McCullough, R. D. Macromolecules 2005, 38, 8649 .

(25) Jeffries-EL, M.; Sauve, G.; McCullough, R. D. Macromolecules 2005, 38,10346

(26) Miyakoshi, R.; Yokoyama, A.; Yokozawa, T. J. Am. Chem. Soc. 2005, 127, 17542

(27) Tamao, K.; Kodama, S.; Nakajima, I.; Kumada, M.; Minato, A.; Suzuki, K. Tetrahedron 1982, 38, 3347.

(28) Martinez-Castro, N.; Lanzendorfer, M. G.; Muller, A. H. E.; Cho, J. C. Acar, M. H.; Faust, R. Macromolecules 2003, 36, 6985.

(29) Jung, M. E.; Blum, R. B. Tetrahedron Lett. 1977, 43, 3791.

JA0733991 